\title{
Optimization Method of Intersection Signal Coordinated Control Based on Vehicle Actuated Model
}

\author{
Chen Zhao-Meng, Liu Xiao-ming, and Wu Wen-Xiang \\ Beijing Key Lab of Urban Traffic Control Technology, North China University of Technology, Beijing 100144, China \\ Correspondence should be addressed to Chen Zhao-Meng; chenzhaomeng@126.com
}

Received 22 July 2014; Revised 22 September 2014; Accepted 25 September 2014

Academic Editor: Wuhong Wang

Copyright (C) 2015 Chen Zhao-Meng et al. This is an open access article distributed under the Creative Commons Attribution License, which permits unrestricted use, distribution, and reproduction in any medium, provided the original work is properly cited.

\begin{abstract}
Traditional timing green wave control with predetermined cycle, split, and offset cannot adapt for dynamic real-time traffic flow. This paper proposes a coordinated control method for variable cycle time green wave bandwidth optimization integrated with traffic-actuated control. In the coordinated control, green split is optimized in real time by the measured presence of arriving and/or standing vehicles in each intersection and simultaneously green waves along arterials are guaranteed. Specifically, the dynamic bound of green wave is firstly determined, and then green early-start and green late-start algorithms are presented respectively to accommodate the fluctuations in vehicle arrival rates in each phase. Numerical examples show that the proposed method improves green time, expands green wave bandwidth, and reduces queuing.
\end{abstract}

\section{Introduction}

Traffic-actuated control is one of traffic signal control modes [1] which does not have a pretimed cycle, signal sequence, and green signal displays and can adapt to the volume of vehicles on the road accordingly. However, because of variable cycle and random light switch, it is practically difficult to apply in the city roads, especially in a coordinated control framework. At present, traffic signal system is still dominated by multiperiod fixed cycle time control in most cities, frequently integrated with green wave control along the city arterials. Green wave control generally attempts to maximize public green wave bandwidth in which a series of traffic lights (usually three or more) are coordinated to allow continuous traffic flow over several intersections in one main direction. Green wave control is popular in signal controls for its simplicity and effectiveness [2], which mainly includes graphical method [3], numerical method [4], and Maxband law [5].

In most green wave optimization models, cycle, split, and offset are generally predetermined and cannot adjust to the real-time fluctuations in traffic, probably lowering its coordination control effectiveness. Therefore, this paper proposes a coordinated control method for variable cycle time green wave bandwidth optimization integrated with traffic-actuated control. The main purpose of this paper is to achieve real-time coordination bandwidth by utilization of green time and actuation of arriving or standing vehicles at each intersection. Furthermore, a numerical example is presented to elaborate our method.

\section{The Key Parameters in Traffic-Actuated Control}

Unlike fixed-cycle traffic signal control, traffic-actuated control has no fixed cycle length, as well as green split, which is dependent on the real-time volume of traffic. In the trafficactuated control, as shown in Figure 1, the process of phase transition is regulated by the following transition rules: (i) when a preset minimum green time from an initial green light ends, the phase changes if there is no vehicle entering in the phase; (ii) otherwise, the phase does not change until the green time is extended to the preset maximum green time or the time when there are no vehicles entering in the phase. The phase transition can effectively reduce vehicle delays and stops and improve the efficiency of the signalized 


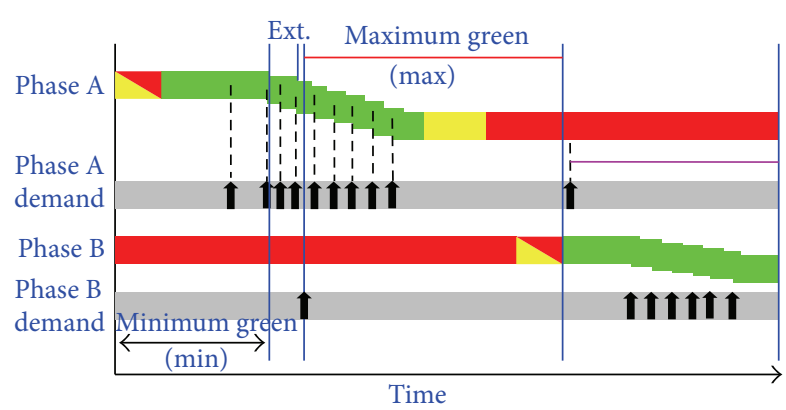

FIgURE 1: Traffic-actuated control.

intersections as long as the maximum green time and unit delaying time are reasonably predetermined.

2.1. Minimum Green Time. Minimum green time represents the least amount of time that a green signal indication will be displayed for a movement. The duration of minimum green is used to allow drivers to react to the start of the green interval and meet driver expectancy and pedestrian crossing time when separate pedestrian signal displays are not provided. The duration of minimum green can also be influenced by detector location. There are various methods for calculating minimum green time, which can be by the types of control system and detector location [6]. In our traffic-actuated control system, detectors are located in the range 2 to 20 meters before stop lines.

The minimum green time is calculated as follows:

$$
\begin{gathered}
G_{\min }=\operatorname{Max}\left[G_{\min v}, G_{\min p}\right], \\
G_{\min v}=S_{\operatorname{lag} v}+h t \times \operatorname{Integer}\left(\frac{L}{S}\right), \\
G_{\min p}=S_{\operatorname{lag} p}+\frac{w}{v_{p}}-t_{g},
\end{gathered}
$$

where $G_{\min }$ is minimum green time in phase; $G_{\min v}$ is minimum green time for vehicles; $G_{\min p}$ is safety time for pedestrian crossing; $S_{\operatorname{lag} v}$ is start-up loss time for vehicles which is commonly set equal to 4 seconds; $h t$ is time headway, equal to 2-3 seconds; $L$ is the distance between the stop line and detector; $S$ is the time (distance) between two adjacent vehicles, equal to 2-3 seconds (5-6 meters); $S_{\operatorname{lag} p}$ is pedestrian start-up loss time, equal to 5 seconds; $w$ is the crossing width for pedestrians; $v_{p}$ is pedestrian crossing speed; $t_{g}$ is green light time interval.

2.2. Maximum Green Time. Maximum green time is the longest green time provided for a phase, which determines whether green time can be effectively utilized, especially when traffic is under moderate congestion level [7, 8]. Unlike traditional fixed maximum green time, the maximum green time proposed in this paper can change according to the fluctuations in volume of traffic in each signal cycle. The maximum green time is given by

$$
\begin{gathered}
G_{\max }=\operatorname{Min}\left[g_{\max }, t_{c}\right], \\
g_{\max }=(C-l) \frac{C V}{C S}, \\
C=\frac{l}{1-C S / R S \times 0.9},
\end{gathered}
$$

where $C V$ is flow rate of a particular phase (vehicles/h); $C S$ is the traffic volume of a particular phase (vehicles/h); $R S$ is the reference traffic flow (vehicles/h); $C$ is cycle length; $R S$ performs a weighting factor of 0.9 , which is designed to operate at $90 \%$ of available capacity; $l$ is loss green time in each cycle.

$g_{\max }$ is the coordination phase value. The number of vehicles getting through the coordination phase is $N_{t v}$ in each cycle. The flow rate is $N_{t v} / S_{v}$, where $S_{v}$ is saturated flow in the lane analyzed.

In order to equilibrate the flow rates of coordinated and noncoordinated phase in one cycle, $g_{\max }$ and $t_{c}$ in noncoordinated phase take the minimum value for calculating the maximum green time. $t_{c}$ is the time when the flow rate reaches $N_{t v} / S_{v}$ in noncoordinated phase. This approach can improve effectiveness of signal intersections, especially when traffic flows are seriously unbalanced in different directions in the noncoordinated phase.

2.3. Unit Extension Time. Unit extension time is the minimum time interval for interrupting the signal between two successive vehicles. This parameter can directly influence right-of-way for vehicles in the queue and vehicle arrivals. If unit extension time is too short, the right-of-way in the phase is not enough, leading to unnecessary delay and stops; if unit extension time is too long, green time is not fully utilized. In addition, to ensure that the detected vehicles can pass the stop line, the distance between detector and stop line and the vehicle speed should be taken into account [9].

In this paper, the distance between detector and stop line is set equal to 20 meters. The minimum average speed is $6 \mathrm{~m} / \mathrm{sec}$. Thus, the unit extension time is not less than 3.3. The flow rate for a lane is 1200 vehicle/h and the average headway is $3600 / 1200$, that is, 3 seconds. Therefore, to multiply a weighting factor to ensure traffic efficiency, unit extension time is given by

$$
\begin{gathered}
e=k_{0} \times \operatorname{Max}\left[p_{t}, h t\right], \\
p_{t}=\frac{L}{v_{i}},
\end{gathered}
$$

where $h t$ is headway, generally equal to $2-3$ seconds, $L$ is the distance between detector and stop line, $v_{i}$ is average speed, $p_{t}$ is the driving time from detector to stop line, and $k_{0}$ is the flat peak time value, generally set equal to 1.1, depending on road traffic characteristics. 

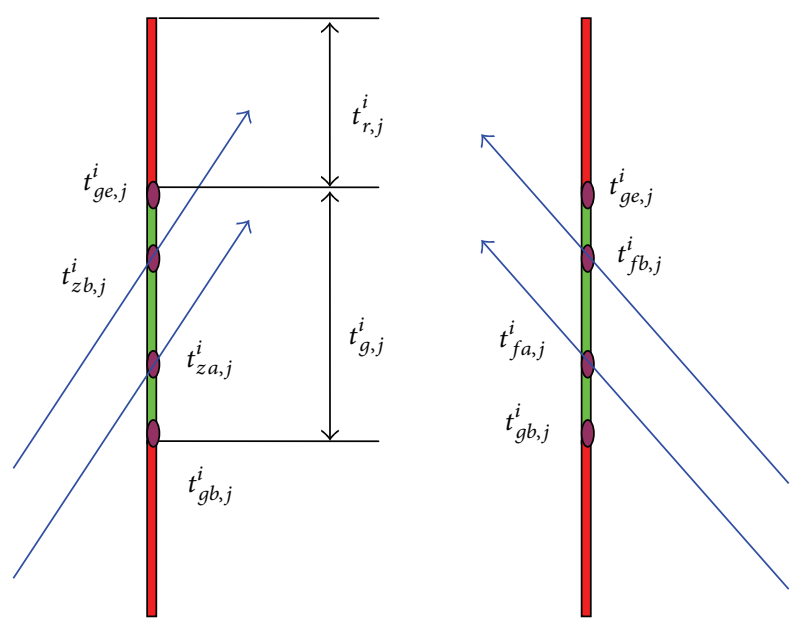

FIGURE 2: Illustration for parameters.

\section{Green Wave Bandwidth}

For convenience, we define the following symbols (as shown in Figure 2). $t_{m, g, j}^{i}$ is the green time in the noncoordinated phase $m$ for the intersection $i$ of cycle $j ; t_{g, j}^{i}$ is the green time in the coordinated phase for intersection $i$ of cycle $j ; t_{g b, j}^{i}$ is the starting green time in the coordinated phase for intersection $i$ of the cycle $j ; t_{g e, j}^{i}$ is the ending green time in the coordinated phase for intersection $i$ of cycle $j ; t_{z a, j}^{i}$ is the starting time of forward green wave in the coordinated phase for intersection $i$ of the cycle $j ; t_{z b, j}^{i}$ is the ending time of forward green wave in the coordinated phase for intersection $i$ of cycle $j ; t_{f a, j}^{i}$ is the starting time of reverse green wave in the coordinated phase for intersection $i$ of cycle $j ; t_{f b, j}^{i}$ is the ending time of reverse green wave in the coordinated phase for intersection $i$ of cycle $j ; t_{\min g, n}^{i}$ is the minimum green time in the phase $n$ for intersection $i$; $t_{\max g, n}$ is the maximum green time in the phase $n$ for intersection $i$ :

$$
\begin{gathered}
t_{\text {begin }, j}^{i}=\min \left(t_{z a, j}^{i}, t_{f a, j}^{i}\right), \\
t_{\text {end }, j}^{i}=\max \left(t_{z b, j}^{i}, t_{f b, j}^{i}\right) .
\end{gathered}
$$

We now investigate green wave bandwidth using graphical method with heuristics. The determination of green wave bandwidth is given as follows [10, 11].

Step 1. Set an identical starting time for each intersection of routes and let $N$ be the number of intersections.

Step 2. Record the starting time $t_{g b, j}^{i}$ and the ending time $t_{g e, j}^{i}$ for each coordination phase in every cycle, and set up $t_{o}^{1}=$ $t_{g b, m}^{1}$ for the coordination phase of the first intersection.

Step 3. Find the initial time $t_{z a, j}^{i}$ for forward green wave bandwidth. If $t_{o}^{2}=t_{g b, m}^{1}+L_{1,2} / V_{z}$ is in the interval $\left[t_{g b, j}^{2}, t_{g e, j}^{2}\right]$ of the second intersection from $t_{o}^{1}$, then judge whether $t_{o}^{3}=$

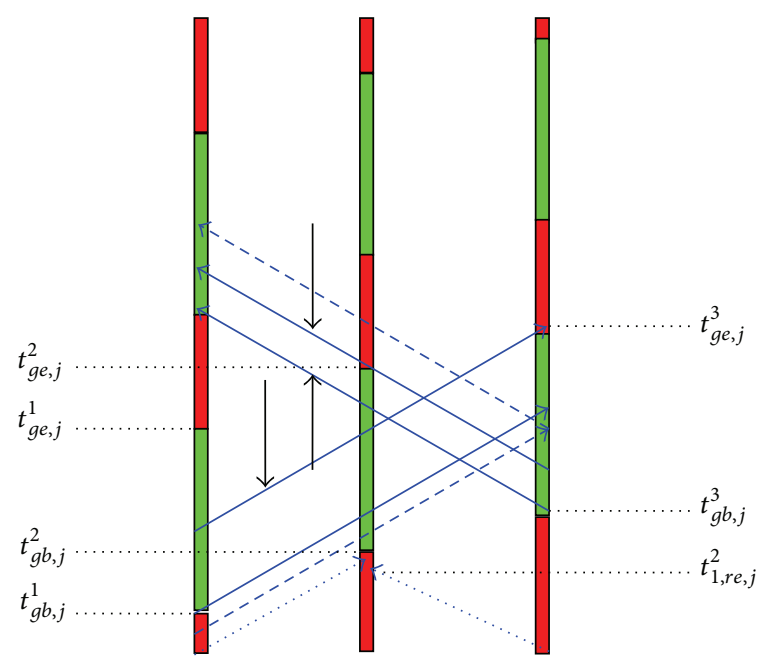

FIGURE 3: The bound of green wave.

$t_{g b, j}^{2}+L_{2,3} / V_{z}$ is in the interval $\left[t_{g b, n}^{i}, t_{g e, n}^{i}\right]$ until the last intersection. If all the conditions are satisfied, then $t_{z a, m}^{1}=t_{o}^{1}$, $t_{z a, j}^{2}=t_{o}^{2}, \ldots, t_{z a, n}^{N}=t_{o}^{N}$, and $t_{o}^{1}=t_{o}^{1}+1$; go to Step 4; otherwise, $t_{o}^{1}=t_{o}^{1}+1$; go back to Step 3 , until $t_{o}^{1}=t_{g e, m}^{1}$.

Step 4. Find the ending time $t_{z b, m}^{i}$ of green wave. If $t_{z a, m}^{i}=$ $t_{g e, m}^{i}$, then green wave bandwidth is 0 ; otherwise, when $t_{o}^{i}=$ $t_{g e, j}^{i}$, set $t_{z b, m}^{1}=t_{o}^{1}, t_{z b, j}^{2}=t_{o}^{2}, \ldots, t_{z b, n}^{N}=t_{o}^{N}$.

We now use the genetic algorithms to derive the maximum green wave bandwidth [12]. To ensure that the bandwidths of both forward and backward directions are consistent with the corresponding traffic flow, the bandwidths yield

$$
\min B=\min \left\{\frac{b_{1}-k b_{2}}{b_{1}+k b_{2}}\right\},
$$

where $b_{1}$ is forward green wave bandwidth; $b_{2}$ is reverse green wave bandwidth; $k$ is unbalanced coefficient of traffic flows of both directions; $B$ is public green wave bandwidth.

\section{The Dynamic Lower Bound of Arterial Green Wave}

The lower bound of arterial green wave is the starting time of an intersection to limit an increase in its bandwidth in forward/reverse direction. As shown in Figure 3, the starting time of forward green wave is the starting time of green light intersection 1 in cycle $j t_{g b, j}^{1}$, which is the lower bound of the forward green wave since it is a binding constraint for forward green wave bandwidth. Similarly, $t_{g b, j}^{3}$ is the lower bound of the reverse green wave.

Under traffic-actuated control, the starting time, the ending time, and the duration for coordination phase of each intersection may vary with volume of traffic, leading to the dynamic lower bounds of both forward and reverse green 


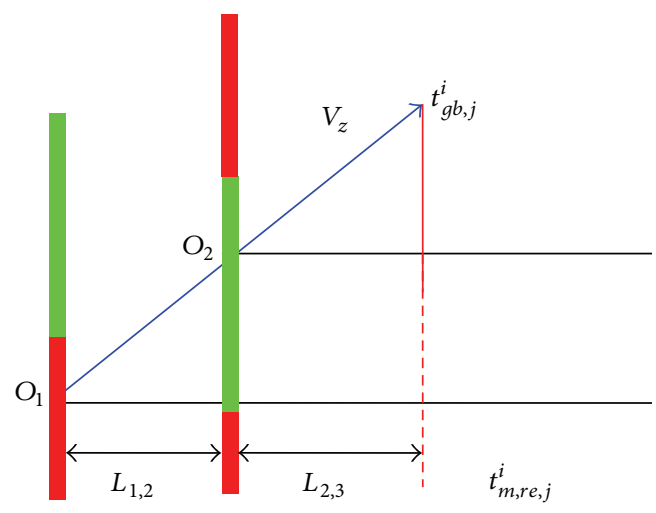

FIGURE 4: The determination of lower bound.

wave bandwidths [13]. The dynamic lower bounds can be obtained as follows.

Step 1. Establish a unified timeline for each intersection of the route and run until the ending green time $t_{m, r e, j}^{i}$ of the noncoordinated phase of target intersection $\left(t_{m, r e, j}^{i}<t_{g b, j}^{i}\right)$. If $t_{m, r e, j}^{i}>t_{g b, j}^{i}$ then go to the control process in Section 6 .

Step 2. From $t_{g b, j}^{i}$, calculate

$$
O_{n}=t_{g b, j}^{i}-\sum_{m=1}^{n} \frac{L_{i, i-m}}{V_{z}} \quad(n=1,2, \ldots, i-1) .
$$

If $t_{m, r e, j}^{i}>O_{i-1}$, find the lower bound for the downstream green wave in each coordinated phase of intersection $1 \sim i$ from $t_{m, r e, j}^{i}$; otherwise, find the lower bound from $O_{i-1}$, as shown in Figure 4. Similarly, for the downstream green wave, calculate $O_{p}=t_{g b, j}^{i}-\sum_{m=i+1}^{n} L_{i, i-m} / V_{f}, n=i+1, i+2, \ldots, N$, where $N$ is the number of the coordination intersections of the route. If $t_{m, r e, j}^{i}>O_{i+1}$, find a lower bound in each coordinated phase of the upstream intersections $i+1 \sim N$ from $t_{m, r e, j}^{i}$; otherwise, find a lower bound from $O_{i+1}$.

Step 3. Judge whether $O_{n}(n=1,2, \ldots, i-1)$ are in $\left[t_{g b, h}^{n}, t_{g e, h}^{n}\right]$. If $t_{g b, h}^{n}<O_{n} \leq t_{g e, h}^{n}$, then $t_{g b, j}^{n}$ is the lower bound of forward green wave; if $t_{g e, h-1}^{n}<O_{n} \leq t_{g b, h}^{n}$, it is not the lower bound. Similarly, find the lower bound of forward green wave.

Step 4. If $t_{g b, j}^{n}$ is neither the lower bound of the forward green wave nor the reverse green wave, then $t_{g b, j}^{n}$ is not the lower bound; otherwise it is the lower bound of the green wave.

\section{Early-Start Algorithm of Green Light for Coordinated Phase}

In the traffic-actuated green wave control, if the running time of noncoordinated phase does not reach the initial design time, the saved green time is assigned to the coordinated phase for obtaining greater green wave bandwidth. Meanwhile, its start time of the green light in the coordinated

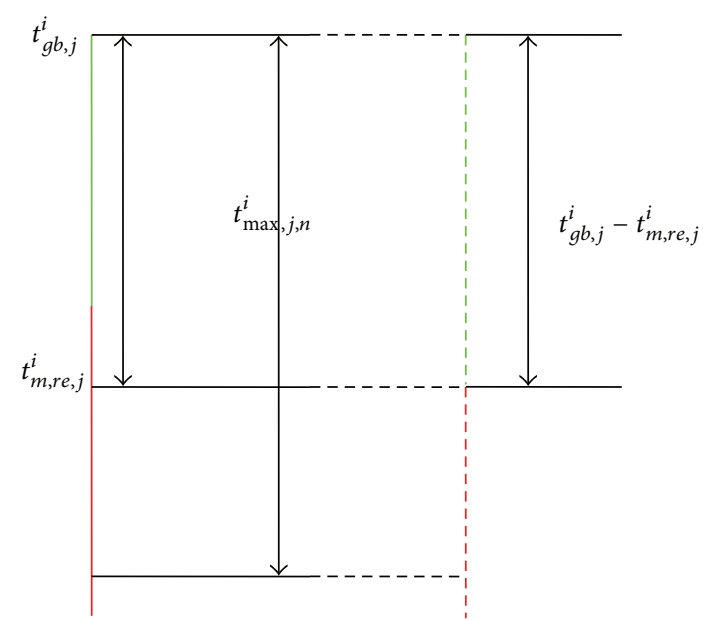

FIgURE 5: The starting time of coordinated phase when $t_{g e, j}^{i}-$ $t_{m, r e, j}^{i} \leq t_{\max j, n}^{i}$.

phase is moved up. However, the phase composition and initial green time allocation of phases in intersections vary with different traffic conditions. Adding the green time of the unused noncoordinated phase to the coordinated may cause such problems as the following: the green time of the coordinated phase is greater than the maximum green time and too early start of the green light in the coordinated phase leads to traffic chaos. Therefore, this paper proposes an earlystart algorithm of green light in the coordinated phase, which is given as follows $[14,15]$.

Step 1. Judge $t_{g e, j}^{i}-t_{m, r e, j}^{i}>t_{\max , n}^{i}$. If it is not true, then replace the starting time by $t_{m, r e, j}^{i}$, as illustrated in Figure 5; otherwise, go to the next step.

Step 2. Judge $t_{\text {end, } j}^{i}-t_{m, r e, j}^{i}>t_{\max , n, n}^{i}$. If it is true, then find $t_{m, r e, j}^{i, \text { up }}$ and let $t_{\text {end,j }}^{i}-t_{m, r e, j}^{i, \text { up }}=t_{\text {max } j, n}^{i}$, and replace the starting time of the green light in the coordinated phase by $t_{m, r e, j}^{i \text {,up }}$ (as shown in Figure 6); if $t_{\text {end,j }}^{i}-t_{m, r e, j}^{i} \leq t_{\max j, n}^{i}$, then go to the next step.

Step 3. Set $t_{o, j}^{i}=t_{g b, j}^{i}$. If $t_{o, j}^{i}$ is the lower bound of green wave, then find $t_{m, r e, j}^{i, \text { up }}$ and set $t_{g e, j}^{i}-t_{m, r e, j}^{i, \text { up }}=t_{\max , n}^{i}$, and the starting time of green light for coordinated phase becomes $t_{m, r e, j}^{i \text {, up }}$ in Figure 7. Otherwise, find the direction of lower bounds (forward green wave or reverse green wave), and then take the forward. Afterwards, take the forward green wave as an example; from $t_{o, j}^{i}$, set $t_{o, j}^{i}=t_{o, j}^{i}-1$, and judge whether $O_{n}$, $n=1,2, \ldots, i-1$, is in $\left[t_{g b, h}^{n}, t_{g e, h}^{n}\right]$. If $t_{g e, h-1}^{n}<O_{n} \leq t_{g b, h}^{n}$, then record the $t_{o, j}^{i}$ which can be regarded as the adjustable virtual bound. Furthermore, compare $t_{m, r e, j}^{i}$ and $t_{o, j}^{i}$. If $t_{o, j}^{i}<t_{m, r e, j}^{i}$, then the starting time of green light for coordinated phase becomes $t_{m, r e, j}^{i}$ and the ending time becomes $t_{m, r e, j}^{i}+t_{\max j, n}^{i}$; if $t_{o, j}^{i}>t_{m, r e, j}^{i}$, when $t_{o, j}^{i}+t_{\max , n}^{i}<t_{g e, j}^{i}$, the starting time of 


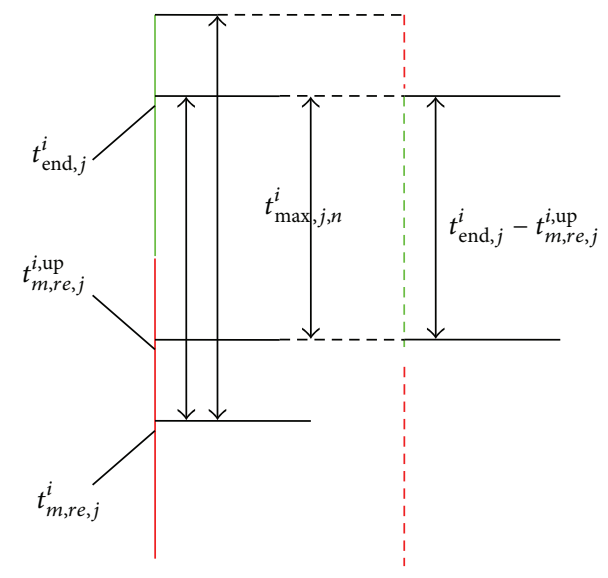

Figure 6: The starting time of coordinated phase when $t_{\text {end, } j}^{i}-$ $t_{m, r e, j}^{i}>t_{\max j, n}^{i}$.

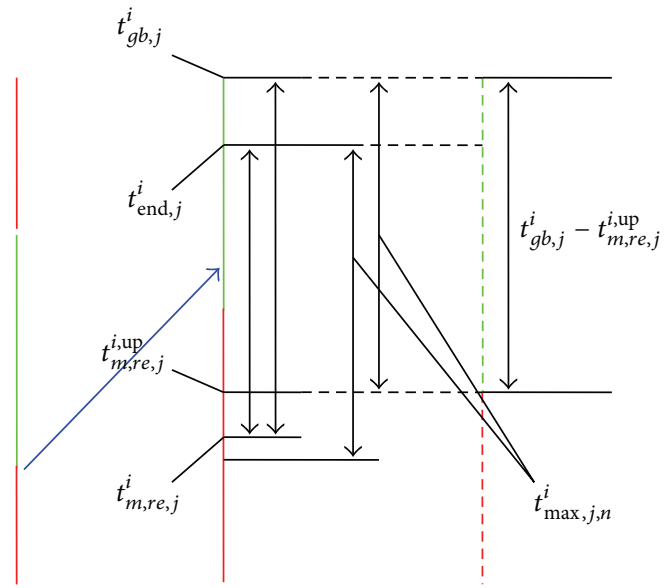

FIgURE 7: The case of starting time of coordinated phase is $t_{m, r e, j}^{i, \text { up }}$.

green light for coordinated phase becomes $t_{o, j}^{i}$ and the ending time becomes $t_{o, j}^{i}+t_{\max j, n}^{i}$, and when $t_{o, j}^{i}+t_{\max j, n}^{i} \geq t_{g e, j}^{i}$, then the starting time is set equal to $t_{g e, j}^{i}-t_{\max j, n}^{i}$ and the ending time becomes $t_{g e, j}^{i}$, as shown in Figure 8 .

\section{Late-Start Algorithm of Green Light for Coordinated Phase}

Due to the randomness and variability of the traffic flow, in terms of the noncoordinated phase in traffic-actuated control, the initial allocation of green time may be lower than the actuated green time. Therefore, it is necessary to extend the green light time to meet the traffic demand in the noncoordinated phase. When the sum of all the green times for noncoordinated phase is larger than the sum of those at the initial allocation time, the start time of green light in coordinated phases will have to be postponed. Accordingly, this paper proposes a late-start algorithm of green light in coordinated phases for determining the maximum expandable capacity, coordinating the traffic capacity of phases after

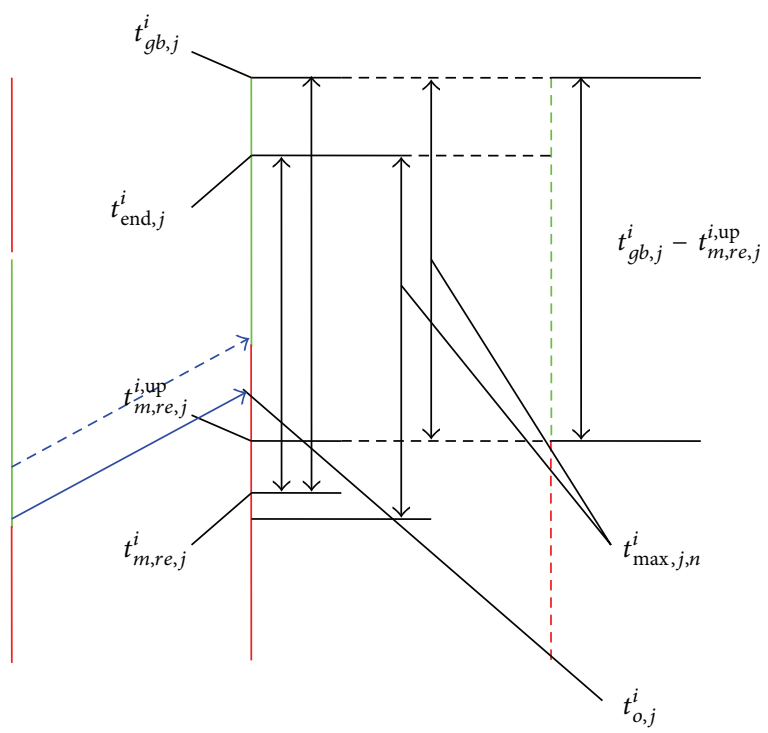

FIGURE 8: The ending time of coordinated phase when $t_{o, j}^{i}+t_{\max j, n}^{i} \geq$ $t_{g e, j}^{i}$.

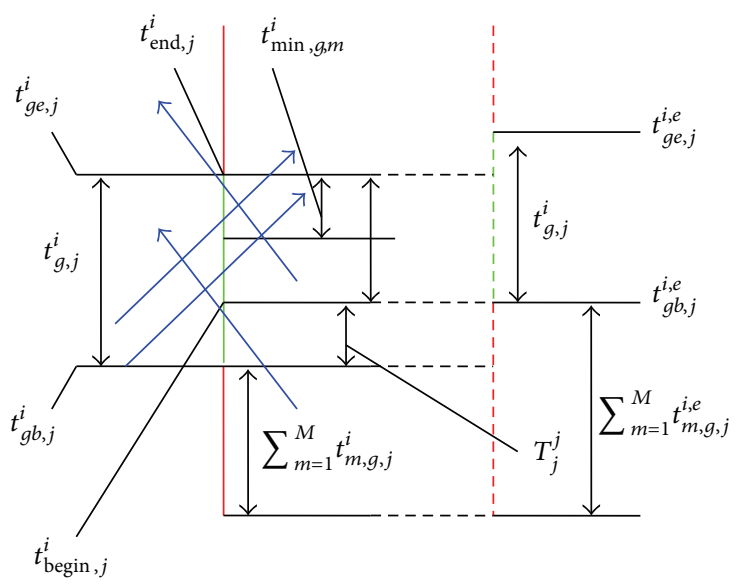

FIgURE 9: The initial maximum expandable capacity of noncoordinated phases when $t_{g e, j}^{i}-t_{\text {begin }, j}^{i} \geq t_{\min g, m}^{i}$.

green delay and assigning green time for coordinated phases under the condition that arterials of green wave bandwidth are satisfied. The algorithm is given in detail as follows $[16,17]$.

Step 1. Determine the initial maximum expandable capacity of noncoordinated phases. With the above method of determining green wave bandwidth, find $t_{\text {begin, } j}^{i}$ and $t_{\text {end, } j}^{i}$ for each intersection in each cycle. If $t_{g e, j}^{i}-t_{\text {begin, } j}^{i} \geq t_{\min g, m}^{i}(m$ denotes a particular coordinated phase), then the maximum expandable capacity can be $T_{j}^{i}=t_{\mathrm{begin}, j}^{i}-t_{g b, j}^{i}$, as shown in Figure 9; otherwise, the maximum expandable capacity is given by $T_{j}^{i}=t_{g e, j}^{i}-t_{\min g, m}^{i}$, as demonstrated in Figure 10 .

Step 2. Determine whether the green time should continue to extend. If $t_{1, g, j}^{i} \geq t_{\min g, 1}^{i}+\Delta t$, then use the above 


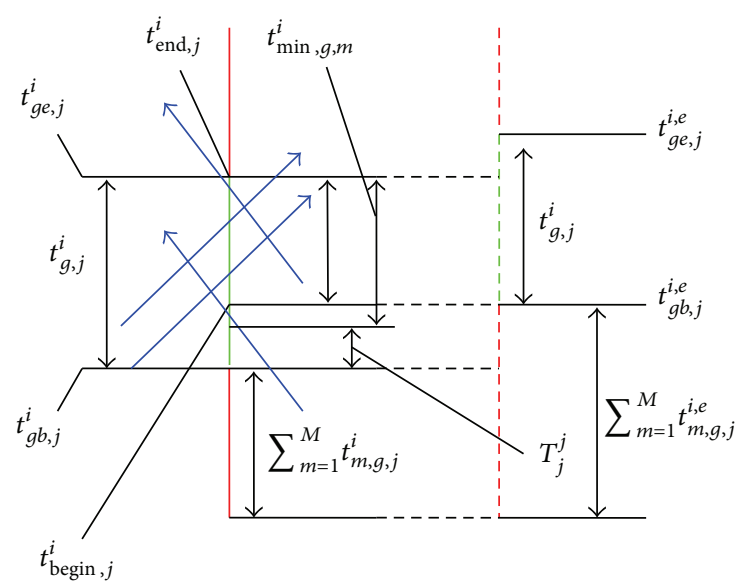

FIGURE 10: The initial maximum expandable capacity of noncoordinated phases when $t_{g e, j}^{i}-t_{\text {begin }, j}^{i}<t_{\min g, m}^{i}$.

method of green wave bandwidth to determine $t_{\text {begin }, j}^{i}, t_{\text {end, } j}^{i}$ and compute $T_{j}^{i}$. If $T_{j}^{i} \geq \Delta t$, then the maximum expandable capacity is modified as $T_{j}^{i}=T_{j}^{i}-\Delta t$; otherwise, continue to determine to extend the green time by $\Delta t$ and modify the maximum expandable capacities $t_{\text {begin, } j}^{i}$ and $t_{\text {end, } j}^{i}$ until it is unnecessary to extend the green time, or $T_{j}^{i}<\Delta t$, or the green time in noncoordinated phase 1 exceeds $t_{\min g, 1}^{i}$.

Step 3. If there are no vehicles in the phase, switch to the next noncoordinated phase in $t_{\min g, 1}^{i}$ and then go back to Step 1. Set $T_{j}^{i}=T_{j}^{i}-\left(t_{1, \text { real }, j}^{i}-t_{1, g, j}^{i}\right)$, where $t_{1, \text { real }, j}^{i}$ is the actual green time for the former noncoordinated phase. If the all noncoordinated phases are subsequently implemented, go to Step 4.

Step 4. Implement the coordinated phase of green lights. If $t_{\text {end, } j}^{i}-t_{\text {end }, j}^{i, f}>t_{\min g, n}^{i}\left(t_{\text {end, } j}^{i, f}\right.$ is the ending green time for the noncoordinated phase), execute traffic-actuated control for coordinated phase from time $t_{\text {end,j }}^{i}$; otherwise, execute from time $t_{\text {end }, j}^{i, f}+t_{\text {min } g, n}^{i}$. To ensure the green wave bandwidth in the following period and the green time for noncoordinated phase, the conditions are given by

$$
\begin{gathered}
t_{\text {end,j }}^{i, x}<t_{\text {end,j } j}^{i, f}+t_{g, j}^{i}, \\
t_{g b, j+1}^{i}-t_{\text {end, } j}^{i, x} \leq \sum_{m=1}^{M} t_{\max g, m}^{i}, \\
t_{g b, j+1}^{i}-t_{\text {end, } j}^{i, x} \geq \sum_{m=1}^{M} t_{\min g, m}^{i},
\end{gathered}
$$

where $M$ is the number of noncoordinated phases. If the ending time for coordinated phase leads to a decrease in the whole green time for the next noncoordinated one, then allocate the whole decreased time to each noncoordinated phase
TABLE 1: The actual traffic signal timing of Guangshun North Street in Wangjing North Road.

\begin{tabular}{lcccccc}
\hline \multirow{2}{*}{ Program } & \multicolumn{7}{c}{ Phase } \\
& Cycle & 1 & 2 & 3 & 4 & Offset \\
\hline Flat peak program & 96 & 42 & 16 & 23 & 15 & 10 \\
High peak program & 140 & 59 & 24 & 38 & 19 & 10 \\
\hline
\end{tabular}

TABLE 2: The actual traffic signal timing of Guangshun North Street in Lize Middle Road.

\begin{tabular}{lcccccc}
\hline \multirow{2}{*}{ Program } & \multicolumn{7}{c}{ Phase } \\
& Cycle & 1 & 2 & 3 & 4 & Offset \\
\hline Flat peak program & 96 & 40 & 14 & 24 & 18 & 25 \\
High peak program & 140 & 60 & 24 & 37 & 19 & 25 \\
\hline
\end{tabular}

TABLE 3: The actual traffic signal timing of Guangshun North Street in Heyin Middle Road.

\begin{tabular}{lcccccc}
\hline \multirow{2}{*}{ Program } & \multicolumn{7}{c}{ Phase } \\
& Cycle & 1 & 2 & 3 & 4 & Offset \\
\hline Flat peak program & 96 & 66 & 30 & & \\
High peak program & 140 & 96 & 44 & & 0 \\
\hline
\end{tabular}

TABLE 4: The actual traffic signal timing of Guangshun North Street in Hongtai West Road.

\begin{tabular}{lcccccc}
\hline \multirow{2}{*}{ Program } & \multicolumn{7}{c}{ Phase } \\
& Cycle & 1 & 2 & 3 & 4 & Offset \\
\hline Flat peak program & 96 & 47 & 15 & 18 & 15 & 25 \\
High peak program & 140 & 70 & 17 & 27 & 26 & 25 \\
\hline
\end{tabular}

and modify the initial green time $t_{m, g, j}^{i}$ for noncoordinated phase of the next cycle.

\section{A Numerical Example}

For eliminating the boundary conditions effect, we only consider four intersections of the arterial in Wangjing zone of Beijing Chaoyang District, which indeed includes six intersections. Specifically, the four coordination-route intersections are located in Guangshun North Street in Wangjing North Road, Guangshun North Street in Lize Middle Road, Guangshun North Street in Heyin Middle Road, and Guangshun North Street in Hongtai West Road respectively. These intersections and the corresponding distribution phase sequence are demonstrated in Figure 11. The current existing traffic signal timings are as shown in Tables $1,2,3$, and 4 . The purpose of the example is to test the effectiveness of the proposed algorithm by comparing with the currently used traffic signal timing $[18,19]$.

Assuming vehicle speed is $40 \mathrm{~km} / \mathrm{h}$ in flat peak period and $25 \mathrm{~km} / \mathrm{h}$ in high peak period, the coordination effect along the arterial from north to south is shown as in Table 5. 


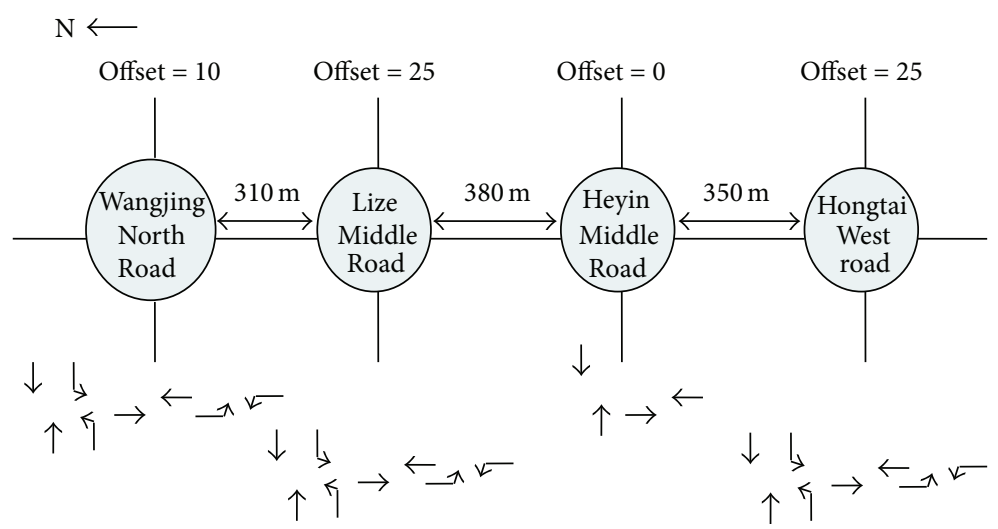

FIGURE 11: Intersection spacing and distribution phase sequence.

TABLE 5: The actual timing bandwidths for 4 intersections.

\begin{tabular}{lcccc}
\hline \multirow{2}{*}{ Period } & \multicolumn{3}{c}{ Bandwidth } \\
& $1-2$ & $2-3$ & $3-4$ & Public bandwidth \\
\hline Flat peak program & 28 & 17 & 35 & 17 \\
High peak program & 31 & 7 & 40 & 7 \\
\hline
\end{tabular}

With VISSIM, we now establish a coordination-route intersection model for simulation in the above four intersections with 20 cycles. The stochastic results are given in Tables $6,7,8,9$, and 10 .

The comparison between the actual green wave bandwidth and the simulated one is illustrated as in Figures 12(a)12(b). In Figures 12(a) and 12(b), 1-2 represents the bandwidth between intersection 1 and intersection 2. Similarly, 2-3 and 3-4 represent the bandwidths between the corresponding two intersections. 1-4 represents the public bandwidth of the four intersections. Clearly, the green wave bandwidth of each intersection in both flat peak and high peak has been greatly improved. The public green wave bandwidths in two periods along the arterial have increased by 7 seconds and 5 seconds, respectively. Therefore, our proposed method is more effective than the original.

Average traffic flows in high peak period and in flat peak period are given in Tables 11 and 12, respectively. With original program the corresponding average queuing vehicles are in Tables 13 and 14, respectively. With the proposed program, the corresponding average queuing vehicles are in Tables 15 and 16 , respectively. Compared with the original program, with the proposed program, the queuing length of coordinated phase for each intersection decreases and approximately equals zero.

\section{Conclusion}

Based on traffic-actuated control and green wave control, this paper has proposed a coordinated control method for variable cycle time green wave bandwidth optimization. In the coordinated control, green split is optimized in real time by the measured presence of arriving and/or standing vehicles in each intersection and simultaneously green waves along
TABLE 6: The simulation traffic signal timing of Guangshun North Street in Wangjing North Road.

\begin{tabular}{lcccccc}
\hline \multirow{2}{*}{ Program } & \multicolumn{7}{c}{ Phase } \\
& Cycle & 1 & 2 & 3 & 4 & Offset \\
\hline Flat peak program & 96 & 49 & 13 & 21 & 13 & 10 \\
High peak program & 140 & 72 & 23 & 30 & 15 & 10 \\
\hline
\end{tabular}

TABLE 7: The simulation traffic signal timing of Guangshun North Street in Lize Middle Road.

\begin{tabular}{lcccccc}
\hline \multirow{2}{*}{ Program } & \multicolumn{7}{c}{ Phase } \\
& Cycle & 1 & 2 & 3 & 4 & Offset \\
\hline Flat peak program & 96 & 44 & 14 & 21 & 17 & 25 \\
High peak program & 140 & 70 & 20 & 32 & 18 & 25 \\
\hline
\end{tabular}

TABLE 8: The simulation traffic signal timing of Guangshun North Street in Heyin Middle Road.

\begin{tabular}{lcccccc}
\hline \multirow{2}{*}{ Program } & \multicolumn{7}{c}{ Phase } \\
& Cycle & 1 & 2 & 3 & 4 & Offset \\
\hline Flat peak program & 96 & 71 & 25 & & & 0 \\
High peak program & 140 & 103 & 37 & & 0 \\
\hline
\end{tabular}

TABLE 9: The simulation traffic signal timing of Guangshun North Street in Hongtai West Road.

\begin{tabular}{lcccccc}
\hline \multirow{2}{*}{ Program } & \multicolumn{7}{c}{ Phase } \\
& Cycle & 1 & 2 & 3 & 4 & Offset \\
\hline Flat peak program & 96 & 53 & 13 & 18 & 12 & 25 \\
High peak program & 140 & 78 & 15 & 26 & 21 & 25 \\
\hline
\end{tabular}

TABLE 10: The simulation using our proposed method for the 4 intersections.

\begin{tabular}{lcccc}
\hline \multirow{2}{*}{ Bandwidth period } & & \multicolumn{3}{c}{ Intersection } \\
& $1-2$ & $2-3$ & $3-4$ & Public bandwidth \\
\hline Flat peak program & 32 & 24 & 41 & 24 \\
High peak program & 41 & 12 & 48 & 12 \\
\hline
\end{tabular}




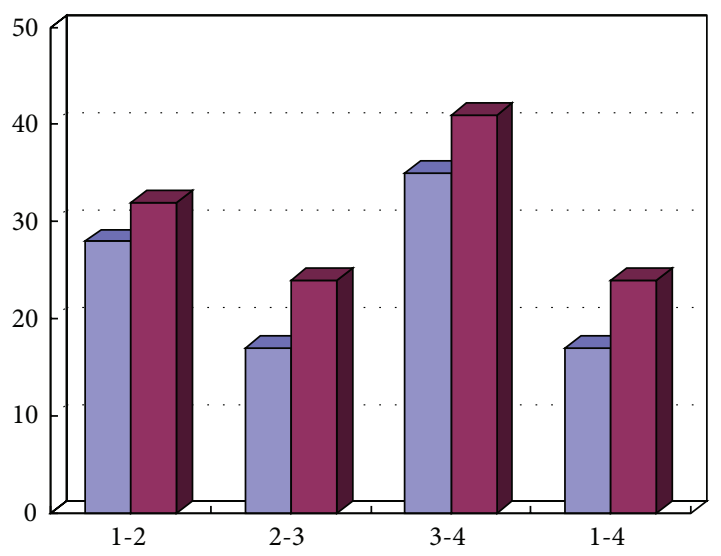

Original method

Proposed method

(a)

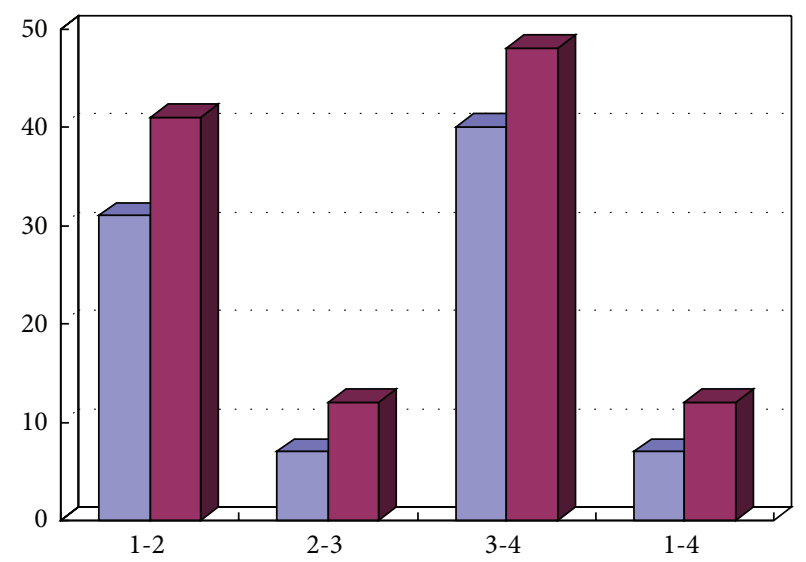

Original method

Proposed method

(b)

FIGURE 12: (a) Green wave bandwidth in flat peak period. (b) Green wave bandwidth in high peak period.

TABLE 11: Average traffic flow data in high peak period (unit: pcu/h).

\begin{tabular}{lcccc}
\hline \multirow{2}{*}{ Phase } & \multicolumn{4}{c}{ Intersection } \\
& 1 & 2 & 3 & 4 \\
\hline PH1 & 1096 & 988 & 890 & 1187 \\
PH2 & 332 & 289 & & 262 \\
PH3 & 476 & 408 & 483 & 367 \\
PH4 & 190 & 168 & & 192 \\
\hline
\end{tabular}

TABLE 12: Average traffic flow data in flat peak period (unit: pcu/h).

\begin{tabular}{lcccc}
\hline \multirow{2}{*}{ Phase } & \multicolumn{4}{c}{ Intersection } \\
& 1 & 2 & 3 & 4 \\
\hline PH1 & 774 & 702 & 679 & 810 \\
PH2 & 260 & 224 & & 242 \\
PH3 & 352 & 316 & 337 & 281 \\
PH4 & 156 & 129 & & 166 \\
\hline
\end{tabular}

TABLE 13: Average queuing vehicles in high peak period by original program (unit: $\mathrm{pcu}$ ).

\begin{tabular}{lllll}
\hline \multirow{2}{*}{ Phase } & \multicolumn{3}{c}{ Intersection } \\
& 1 & 2 & 3 & 4 \\
\hline PH1 & 9 & 5 & 0 & 7 \\
PH2 & 0 & 0 & & 2 \\
PH3 & 4 & 0 & 0 & 0 \\
PH4 & 0 & 3 & & 1 \\
\hline
\end{tabular}

arterials are guaranteed. Specifically, the dynamic bound of green wave is firstly determined, and then green early-start and green late-start algorithms are presented respectively to accommodate the fluctuations in vehicle arrival rates in each phase.
TABLE 14: Average queuing vehicles in flat peak period by original program (unit: $\mathrm{pcu}$ ).

\begin{tabular}{lllll}
\hline \multirow{2}{*}{ Phase } & \multicolumn{3}{c}{ Intersection } \\
& 1 & 2 & 3 & 4 \\
\hline PH1 & 6 & 3 & 0 & 6 \\
PH2 & 2 & 0 & & 1 \\
PH3 & 3 & 4 & 0 & 0 \\
PH4 & 0 & 1 & & 2 \\
\hline
\end{tabular}

TABLE 15: Average queuing vehicles in high peak period by present program (unit: pcu).

\begin{tabular}{lllll}
\hline \multirow{2}{*}{ Phase } & \multicolumn{3}{c}{ Intersection } \\
& 1 & 2 & 3 & 4 \\
\hline PH1 & 4 & 1 & 0 & 3 \\
PH2 & 1 & 1 & & 2 \\
PH3 & 2 & 0 & 0 & 1 \\
PH4 & 0 & 1 & & 0 \\
\hline
\end{tabular}

TABLE 16: Average queuing vehicles in high peak period by present program (unit: $\mathrm{pcu}$ ).

\begin{tabular}{lllll}
\hline \multirow{2}{*}{ Phase } & \multicolumn{3}{c}{ Intersection } \\
& 1 & 2 & 3 & 4 \\
\hline PH1 & 3 & 2 & 0 & 4 \\
PH2 & 0 & 1 & & 0 \\
PH3 & 0 & 0 & 0 & 1 \\
PH4 & 1 & 0 & & 0 \\
\hline
\end{tabular}

Finally, we have used a real four-intersection arterial in Wangjing zone of Beijing Chaoyang District to test our proposed model. Comparing the original method and our proposed method, we find that the proposed method improves green time, expands green wave bandwidth, and 
reduces queuing, consequently leading to an increase in the road network efficiency.

\section{Conflict of Interests}

The authors declare that there is no conflict of interests regarding the publication of this paper.

\section{Acknowledgments}

This research was supported by grants from the National Natural Science Foundation of China (71071004, 71271004, and 71471002).

\section{References}

[1] L. J. Pignataro, W. R. McShane, and K. W. Crowley, Traffic Control in Oversaturated Street Networks, Transportation Research Board, Washington, DC, USA, 1978.

[2] S. H. Fan and H. B. Ding, "One-way green-wave research," in Proceedings of the International Conference on Remote Sensing, Environment and Transportation Engineering (RSETE '11), pp. 6133-6135, June 2011.

[3] R. L. Gordon, "A technique for control of traffic at critical intersections," Transportation Science, vol. 3, no. 4, pp. 279-288, 1969.

[4] A. K. Rathi, "A control scheme for high traffic density sectors," Transportation Research B, vol. 22, no. 2, pp. 81-101, 1988.

[5] K. Lu and J. M. Xu, "Offset model for arterial road coordinate control and its optimization method," China Journal of Highway and Transport, vol. 21, no. 1, pp. 83-88, 2008 (Chinese).

[6] G. H. Zhang and Y. H. Wang, "Optimizing minimum and maximum green time settings for traffic actuated control at isolated intersections," IEEE Transactions on Intelligent Transportation Systems, vol. 12, no. 1, pp. 164-173, 2011.

[7] W. Guo, Z. Yu, Z. He, and S. Zeng, "Traffic-actuated signal control based on dynamic optimal maximum green time," in Proceedings of the International Conference on Transportation Engineering (ICTE '07), pp. 582-587, July 2007.

[8] S. M. Click and A. Rajagopalan, "Evaluation of variable maximum green time to improve rural traffic signal operations," Transportation Research Record, vol. 2080, pp. 75-83, 2008.

[9] J. Cai, H. Y. Liu, L. H. Zhang, and Z. Wang, "The optimal unit green extension-considering different demand patterns," Applied Mechanics and Materials, vol. 409-410, pp. 1357-1365, 2013.

[10] X. M. Liu and L. Wang, "Optimization method of intersection signal coordinated control based on integrated green wave bandwidth maximization," Journal of Jilin University (Engineering and Technology Edition), vol. 43, no. 1, pp. 62-67, 2013 (Chinese).

[11] L. Y. Gao, X. J. Hu, W. Wang, and S. S. Yu, "Development and evaluation of a green wave control algorithm based on two-way bandwidth maximization for transit signal priority," Applied Mechanics and Materials, vol. 505-506, pp. 1046-1054, 2014.

[12] S. Lv, C. Li, Z. Li, and Q. Zang, "Coordinate signal control in urban traffic of two-direction green wave based on genetic BP neural network," Advanced Materials Research, vol. 823, pp. 665-668, 2013.
[13] A. de Abreu Batista and L. R. Coutinho, "A multiagent system for combining green wave and adaptive control in a dynamic way," in Proceedings of the IEEE 16th International Conference on Intelligent Transportation Systems (ITSC '13), pp. 2439-2444, The Hague, The Netherlands, October 2013.

[14] J. G. Wang, L. Wang, Q. Zhang, Y. Z. Li, X. T. Meng, and N. Y. Liu, "Phase optimization of arterial coordinated control," in Proceedings of the 2nd International Conference on Transportation Engineering (ICTE '09), pp. 2552-2559, July 2009.

[15] E. C. P. Chang and J. Koothrappally, "Field verification of coordinated actuated control," Transportation Research Record, no. 1456, pp. 83-90, 1994.

[16] C. Y. Mao and Y. L. Pei, "Phase and timing optimization at actuated-coordinated signal control intersection," in Proceedings of the 9th International Conference of Chinese Transportation Professionals (ICCTP '09), 2009.

[17] X. Sun, T. Urbanik, and L. D. Han, "Neurofuzzy control to actuated-coordinated system at closely-spaced intersections," Applied Mechanics and Materials, vol. 321-324, pp. 1249-1258, 2013.

[18] D. E. Lucas, P. B. Mirchandani, and K. L. Head, "Remote simulation to evaluate real-time traffic control strategies," Transportation Research Record, no. 1727, pp. 95-100, 2000.

[19] J. Mikalauskas, “Traffic control system simulation," Transport Means-Proceedings of the International Conference, pp. 41-44, 2010. 


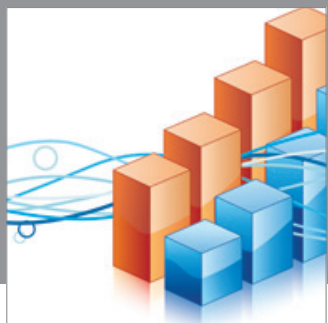

Advances in

Operations Research

mansans

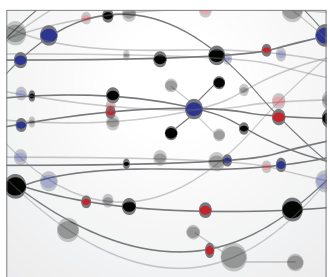

The Scientific World Journal
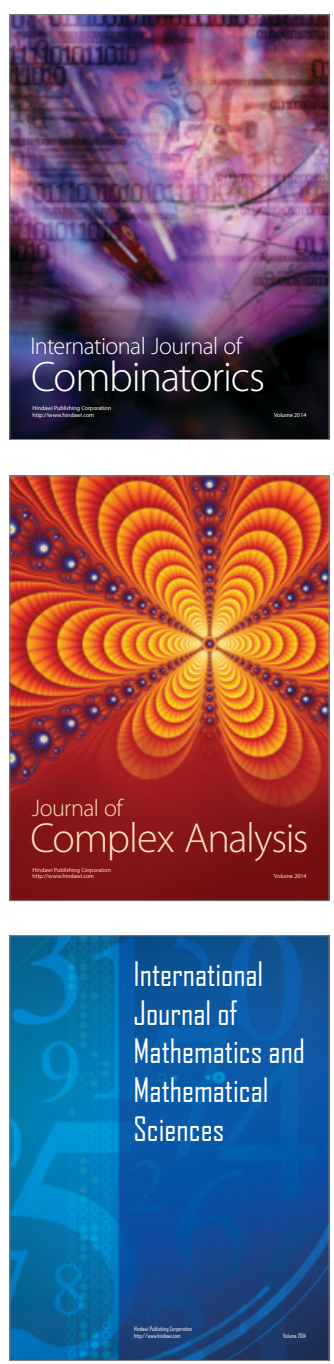
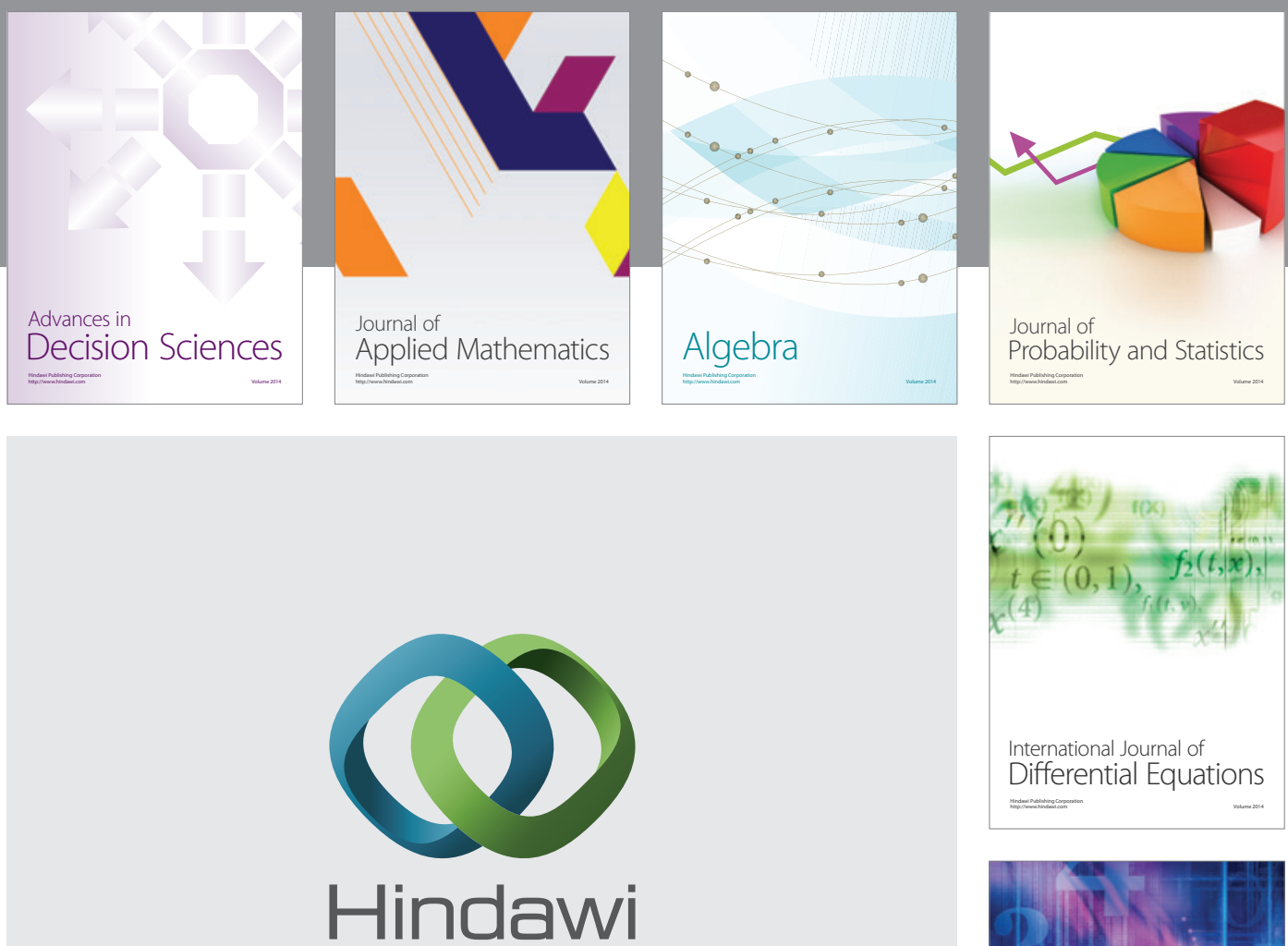

Submit your manuscripts at http://www.hindawi.com
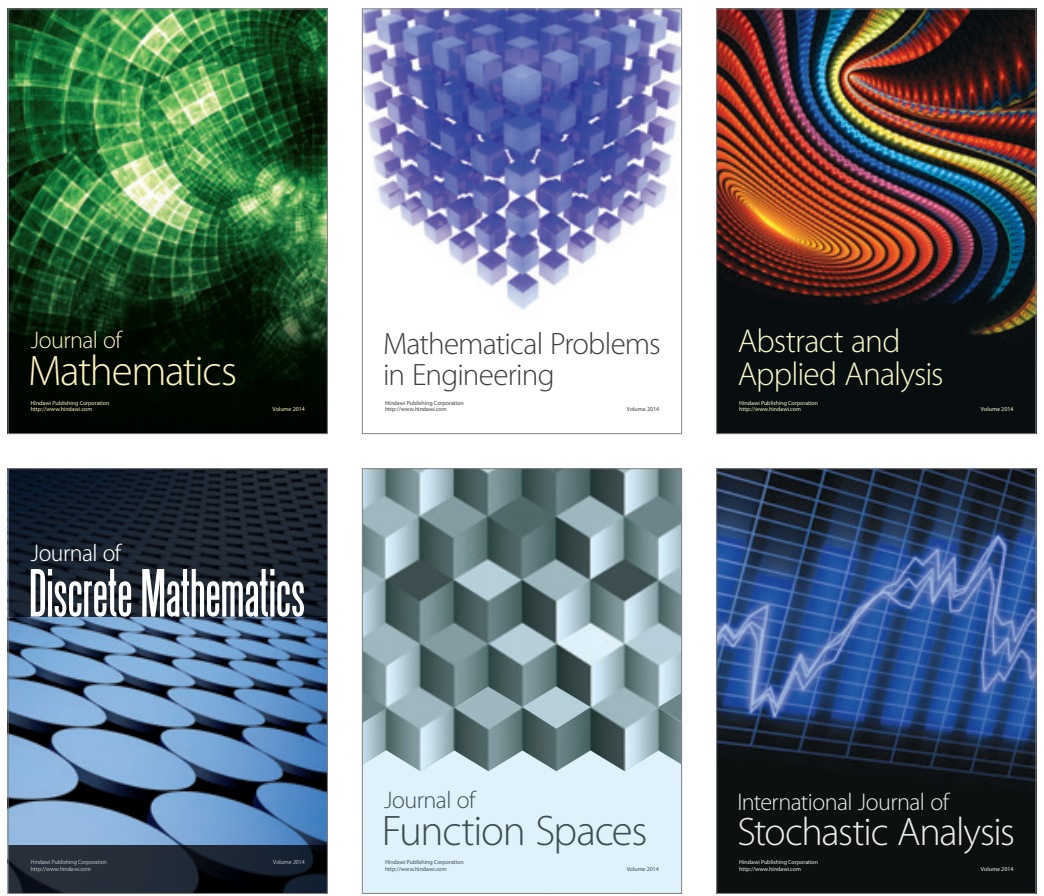

Journal of

Function Spaces

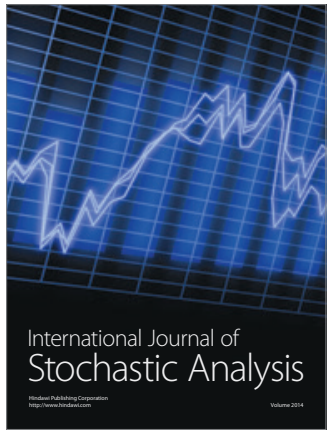

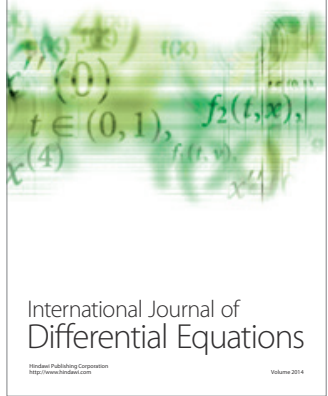
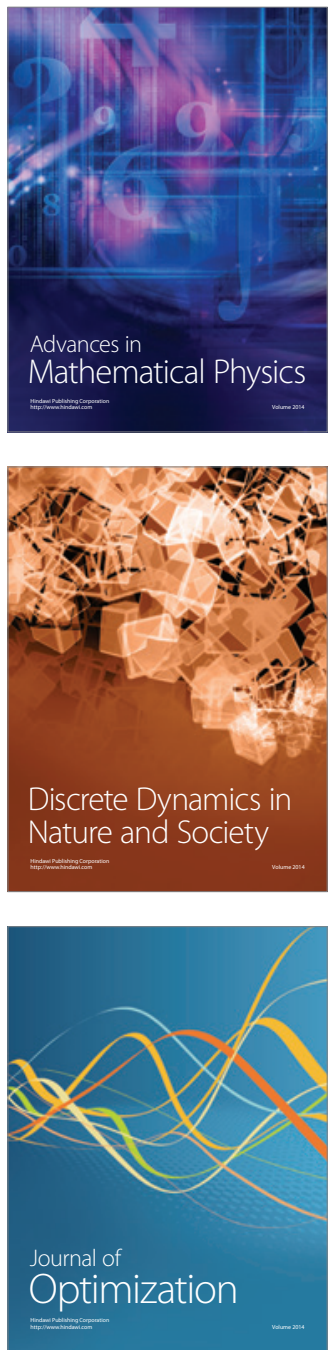